Research, Society and Development, v. 9, n. 10, e7019109016, 2020

(CC BY 4.0) | ISSN 2525-3409 | DOI: http://dx.doi.org/10.33448/rsd-v9i10.9016

\title{
Sistemas silvipastoris no Brasil: uma revisão sistemática
}

Silvopastoral systems in Brazil: a systematic review

Sistemas silvipastorales en Brasil: una revisión sistemática

Recebido: 04/10/2020 | Revisado: 11/10/2020 | Aceito: 13/10/2020 | Publicado: 15/10/2020

\section{Giovana Pittarelli Bento}

ORCID: https://orcid.org/0000-0002-8401-2361

Universidade Federal de Santa Catarina, Brasil

E-mail: giovanapitta@ hotmail.com

Abdon Luiz Schmitt Filho

ORCID: https://orcid.org/0000-0002-3553-7727

Universidade Federal de Santa Catarina, Brasil

E-mail: abdonfilho@hotmail.com

Márcia Regina Faita

ORCID: https://orcid.org/0000-0003-1664-134X

Universidade Federal de Santa Catarina, Brasil

E-mail: marcia.faita@gmail.com

\section{Resumo}

Esta pesquisa tem como objetivo apresentar um histórico dos avanços e da diversidade de dispersão dos Sistemas Silvipastoris no Brasil, através de informações disponíveis na literatura científica. Utilizou-se como método de pesquisa a revisão sistemática da literatura, usando a base de dados Web of Science. No total, 63 artigos foram selecionados com base em critérios de inclusão e exclusão, de uma amostragem inicial de 374. A análise dos artigos selecionados permitiu identificar 73 sistemas silvipastoris, distribuídos por todas as regiões geopolíticas brasileiras. Os sistemas foram categorizados de acordo com a região de implantação, a diversidade e os objetivos dos estudos, relacionando os componentes animais, pastagem, arbóreo, econômico e silvipastoril como sistema. Por fim, essa revisão sistemática permitiu o desenvolvimento de um mapa de evidências, demonstrando as lacunas de conhecimento sobre o tema, bem como as necessidades de avanço nos estudos.

Palavras-chave: Agroflorestas; Arborização de pastagem; Pastagem; Pecuária de baixo $\sim$ carbono; Sombreamento de pastagem. 


\begin{abstract}
This research aims to provide a history of advances and the diversity of dispersion of Silvipastoral Systems SPS in Brazil based scientific literature. The method used comprised a systematic literature review, using the Web of Science database. In total, 63 articles were selected based on inclusion and exclusion criteria, from an initial sample of 374 . The analysis of the selected articles allowed the identification of 73 silvopastoral systems, distributed across all Brazilian geopolitical regions. The SPS were categorized according to the region, diversity and objectives of the studies. These objectives were animal based studies, pasture, tree, economic or SPS components. Finally, this systematic review allowed the development of an evidence map, demonstrating the knowledge gaps on the topic, and the need for further studies.
\end{abstract}

Keywords: Agroforestry; Low carbon livestock; Pasture shading; Pasture; Trees on pasture.

\title{
Resumen
}

Esta investigación tiene como objetivo proporcionar una historia de los avances y la diversidad de dispersión de los Sistemas Silvopastoriles SSP en Brasil, a través de la literatura científica. El método utilizado consistió en una revisión sistemática de la literatura, utilizando la base de datos Web of Science. En total, se seleccionaron 63 artículos con base en criterios de inclusión y exclusión, de una muestra inicial de 374. El análisis de los artículos seleccionados permitió identificar 73 sistemas silvopastoriles, distribuidos en todas las regiones geopolíticas brasileñas. Los sistemas se categorizaron según la región de implantación, la diversidad y los objetivos de los estudios. Estes eram estudios relacionando los componentes animales, pastizal, arbóreo, económico y componentes del SSP. Finalmente, esta revisión sistemática permitió la elaboración de un mapa de evidencia, demostrando las brechas de conocimiento sobre el tema y la necesidad de avanzar en los estudios.

Palabras clave: Agroforestería; Forestación de pastos; Ganado bajo en carbono; Sombreado de pastos; Praderas.

\section{Introdução}

Um dos maiores desafios da pesquisa agropecuária brasileira é manter a produtividade, impedindo a continua degradação do meio ambiente pela agricultura convencional (Abadias et al., 2020; Paciullo et al., 2014). Na busca de atender esta demanda, os sistemas integrados de produção, como os sistemas silvipastoris (SSP) têm despertado a 
atenção de produtores e pesquisadores (Furtado et al., 2019; Joseph et al.; 2019; Schmitt Filho e Farley, 2020). Estes sistemas apresentam práticas agroflorestais integradas simultaneamente à pecuária, usando tanto espécies florestais nativas como exóticas (Peri et al., 2016).

As árvores proporcionam redução da temperatura do ar, o aumento da umidade relativa no entorno e, com a sombra projetada, melhoram o microclima local (Deniz et al., 2018; Kretzer, 2019) e o bem-estar do rebanho (Broom, 2017; Deniz et al. 2020). Os serviços ecossistêmicos (SE) globais e regionais dos sistemas silvipastoris (SSP) são aditivos aos SE locais e a multifuncionalidade da paisagem (Huntsinger et al., 2004). Estes sistemas são reconhecidamente importantes para a transição da produção pecuária tradicional extensiva, para produção sustentável (Alves et al., 2015).

Para que o SSP seja eficiente, é necessário que a escolha de espécies arbóreas, pastagens e animais, priorizem indivíduos adaptados às características dos agroecossistemas locais. As diferenças nos SSPs podem ser atribuídas às peculiaridades regionais dos biomas, dos ecossistemas, das propriedades e das condições socioeconômicas e culturais dos agricultores (Balbino et al., 2011). Diante deste contexto, o presente trabalho teve como objetivos apresentar um histórico dos avanços e da diversidade de dispersão dos SSPs no Brasil, através de uma revisão sistemática da literatura científica.

\section{Metodologia}

A revisão sistemática reúne e sintetiza resultados de estudos primários individuais já existentes (Collaboration for environmental evidence, 2013). São utilizadas para responder perguntas, testar hipóteses, criar generalizações sobre o tema e demonstrar as lacunas de pesquisa (Cooper e Hedges, 2009). Nesta revisão sistemática, foram seguidos os passos estabelecidos pela Collaboration for Environmental Evidence - CEE (2013), representados abaixo (Figura 1). 
Figura 1. Estrutura da revisão Sistemática.

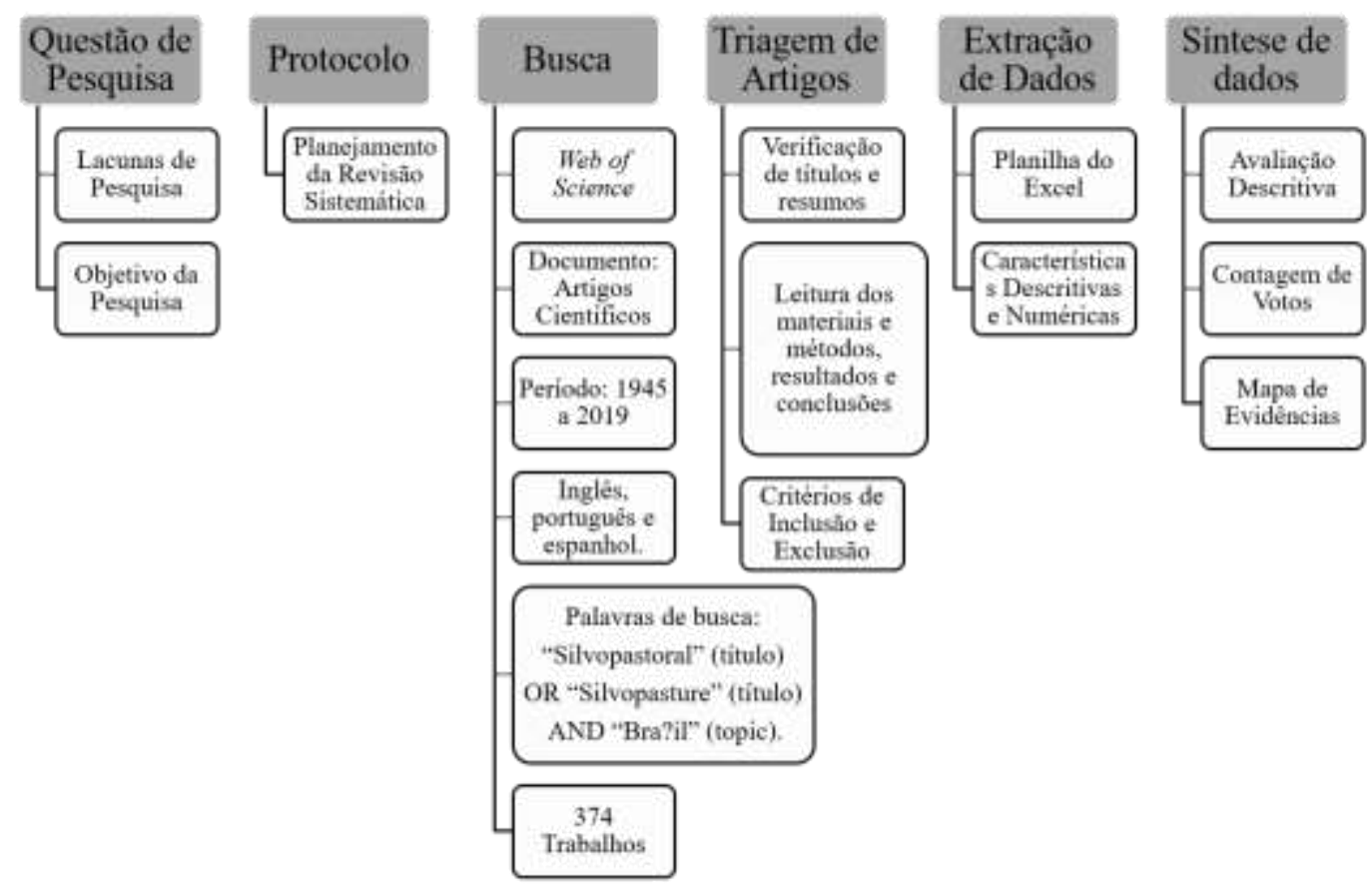

Fonte: Elaborado pelos autores.

No diagrama acima é possível observar as etapas do planejamento com a elaboração da questão de pesquisa, e retirada das lacunas da literatura. Na fase da pesquisa, a busca por artigos foi realizada com a seleção das bases de dados e filtros de inclusão e exclusão. Em seguida iniciou-se a triagem e a seleção dos artigos com foco no objetivo da pesquisa e análise de conteúdo.

\subsection{Busca de dados}

Inicialmente, a busca de informações sobre estudos científicos que já foram conduzidos no Brasil sobre SSPs foi realizada na base Web of Science (coleção principal, Clarivate Analytics), considerando os anos entre 1945 e 2019. Os trabalhos selecionados foram do tipo artigo científico e revisões de literatura, limitados aos idiomas inglês, português e espanhol.

A heurística foi definida da seguinte maneira: Estratégia de busca, com a utilização dos termos "Silvopastoral" (título) OR "Silvopasture" (título) AND "Bra?il" (topic). O termo "Bra?il" poderia estar contido no título, resumo ou palavras-chave dos trabalhos, enquanto as demais somente no título dos trabalhos. 
Research, Society and Development, v. 9, n. 10, e7019109016, 2020

(CC BY 4.0) | ISSN 2525-3409 | DOI: http://dx.doi.org/10.33448/rsd-v9i10.9016

De acordo com o critério de busca, foram encontrados 374 trabalhos, destes 321 estavam em inglês, 28 em espanhol e 23 em português.

\subsection{Triagem dos artigos}

A triagem dos artigos resultantes da busca na Web of Science foi realizada em duas etapas. Primeiramente, através da verificação de títulos e resumos, sendo eliminados aqueles que não se encaixavam nos critérios (Tabela 1). Na segunda etapa, foi realizada a leitura dos materiais e métodos, resultados e conclusões de cada trabalho, de modo a restringir a área de abrangência da pesquisa no território brasileiro.

Os critérios foram construídos para gerar uma triagem de fontes adequadas aos objetivos da revisão. Foi estabelecida uma definição de SSP passível de ser analisada com as informações dos artigos científicos.

Tabela 1. Critérios de inclusão e exclusão utilizados na triagem de fontes adequada aos objetivos da revisão.

\begin{tabular}{cc}
\hline Inclui & Exclui \\
\hline $\begin{array}{c}\text { SSP que integram pecuária, } \\
\text { componentes florestais e pastagens }\end{array}$ & $\begin{array}{c}\text { Sistemas que não estavam } \\
\text { implantados dentro do } \\
\text { território Brasileiro. }\end{array}$ \\
\hline $\begin{array}{c}\text { Sistemas com no mínimo 1 espécie } \\
\text { lenhosa ou herbácea de médio } \\
\text { porte. }\end{array}$ \\
\hline $\begin{array}{c}\text { SSP com as espécies arbóreas citadas no } \\
\text { trabalho }\end{array}$ \\
\hline
\end{tabular}

Fonte: Elaborado pelos autores.

Nota-se na Tabela 1, que três critérios de inclusão e um critério de exclusão foram utilizados durante a fase de triagem dos artigos enquadrando-os aos objetivos desta revisão.

Dos 374 artigos encontrados, apenas 63 foram selecionados após a etapa de triagem, pois satisfizeram os critérios de seleção, descrevendo 73 SSPs dispersos pelo território nacional. Os artigos encontrados apresentam uma cronologia de 1994 a 2019, contribuindo na compreensão dos avanços da silvicultura Brasileira, e no desenvolvimento de sistemas de produção integrados, até o sistema silvipastoril. 


\subsection{Extração e síntese de dados}

Para a extração dos dados, foi elaborada uma planilha de dados contendo colunas para inclusão das características descritivas e numéricas dos estudos. Os critérios utilizados para a categorização dos sistemas silvipastoris foram divididos em etapas. Desse modo, todas as variáveis identificadas foram classificadas em categorias sociopolíticas e de diversidade. Para facilitar compreensão, os dados são apresentados em gráficos e em um mapa de evidências.

\section{Resultados e Discussão}

\subsection{Sistemas silvipastoris e silvicultura}

Durante a década de 60, com a extração da madeira dos remanescentes florestais, alguns biomas brasileiros tiveram suas áreas extremamente degradadas. Já em meados da década de 70, com o estabelecimento da crise energética, a madeira passava a ser a fonte energética do setor industrial e doméstico, incentivando os plantios das monoculturas arbóreas, também denominados de reflorestamentos. A criação de institutos estaduais, que regulamentavam e incentivavam a pesquisa "florestal", proporcionaram a introdução de espécies exóticas para este fim que, devido ao bom desenvolvimento, se distribuíram rapidamente por todos os biomas brasileiros (Silva, 2016).

$\mathrm{Na}$ década de 70, período marcado pelo aumento do interesse empresarial na silvicultura, surge um maior número de profissionais ligados a estas atividades, promovendo a evolução da "ciência florestal" ou silvicultura no país. Neste período, ocorreu uma franca expansão das monoculturas arbóreas de espécies exóticas, notadamente pinus e eucaliptos (Antonangelo \& Bacha, 1998). Neste período a pecuária extensiva em pastagens destituídas de árvores também se expandia, impulsionando grandes desmatamentos e originado uma série de externalidades ambientais. O panorama de degradação levou a comunidade científica buscar sistemas integrados e mais sustentáveis, para harmonizar o aumento de produtividade vegetal e animal, com a preservação de recursos naturais (Balbino et al., 2011).

Dentre os sistemas integrados, os SSPs vêm sendo amplamente difundidos nos trabalhos realizados pela Empresa Brasileira de Pesquisa Agropecuária (EMBRAPA), responsável pelas primeiras propostas de sistemas integrados de produção do país, com estudos na área desde a década de 1980 (Schreiner, 1983). No final dos anos 1990 o tema se 
(CC BY 4.0) | ISSN 2525-3409 | DOI: http://dx.doi.org/10.33448/rsd-v9i10.9016

solidificou como alternativa à pecuária extensiva convencional, destituída dos elementos agroflorestais ou florestais (Balbino et al., 2011)

Em 2013 os SSPs foram contemplados pela Lei 12.805/2013, sancionada em 30 de abril daquele ano, instituindo a Política Nacional de Integração Lavoura-Pecuária-Floresta. Esta tem como princípio melhorar de forma sustentável a produtividade, qualidade e a renda das atividades agropecuárias através de sistemas integrados de produção (Alves et al., 2015) Isto gerou uma popularização do tema, atraindo instituições de pesquisa e empresas privadas, para fomentar as ações em todo território nacional (Saibro \& Garcia, 2005). Na Figura 2, vemos as publicações dentro do contexto histórico indicado.

Figura 2. Artigos científicos publicados entre os anos de 1945 e 2019, selecionados para compor este estudo por apresentar os termos "Silvipastoril" no título, e "Brasil" no título e palavras-chave, nos idiomas português, inglês e espanhol.

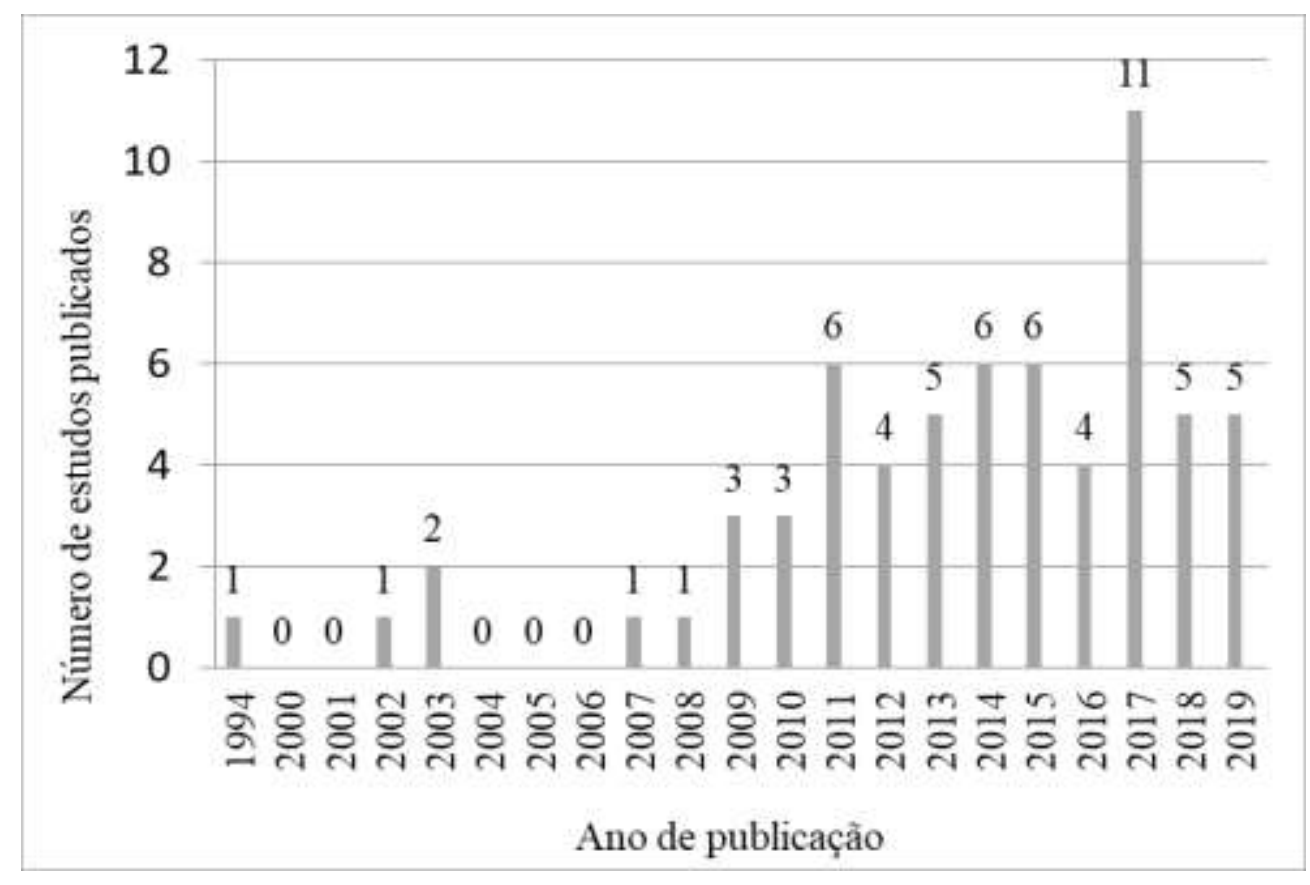

Fonte: Elaborado pelos autores.

Na Figura 2 observa-se que o inicio do fluxo de artigos relacionados aos sistemas silvipastoris ocorreu na década de 90. Entretanto a intensificação deste tipo de publicação acontece recentemente, em 2011. 


\subsection{Distribuição geográfica dos sistemas silvipastoris}

Balbino et al. (2011) apontaram que a Integração Lavoura-Pecuária-Floresta em suas diferentes modalidades poderia, de forma geral, ser estimada em 1,6 milhões de hectares no território nacional. Não existem estatísticas precisas e oficiais referentes a adoção dos SSPs no Brasil (Wruck et al., 2015). Os artigos analisados evidenciam um cenário de dispersão dos SSPs em 14 estados brasileiros, além do Distrito Federal (Figura 3).

Figura 3. Número de SSPs identificados nos artigos científicos publicados entre os anos de 1945 e 2019, por estados brasileiros.

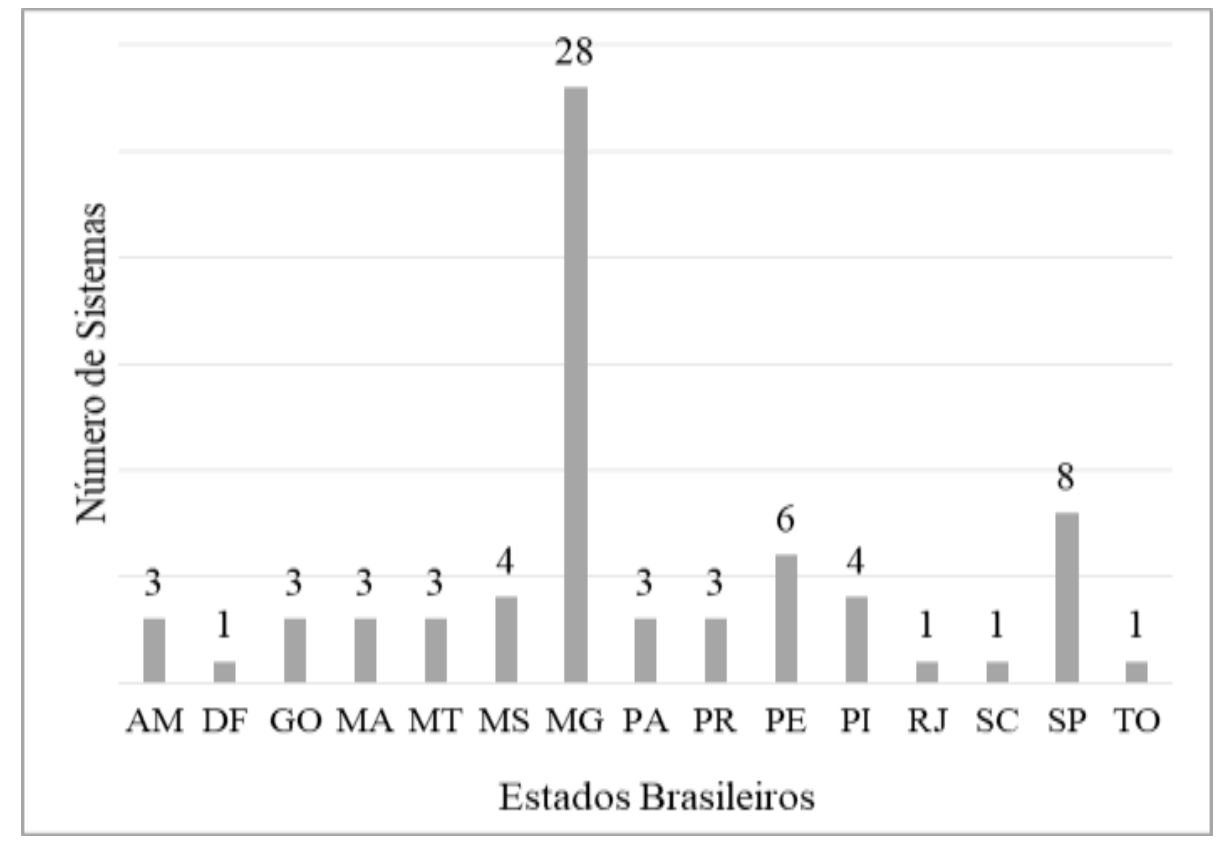

Fonte: Elaborado pelos autores.

Na Figura 3 observa-se a maioria dos estudos em Minas Gerais, São Paulo e Pernambuco. 
Figura 4. Número de SSPs identificados nos artigos científicos publicados entre os anos de 1945 e 2019, por região geográfica brasileira.

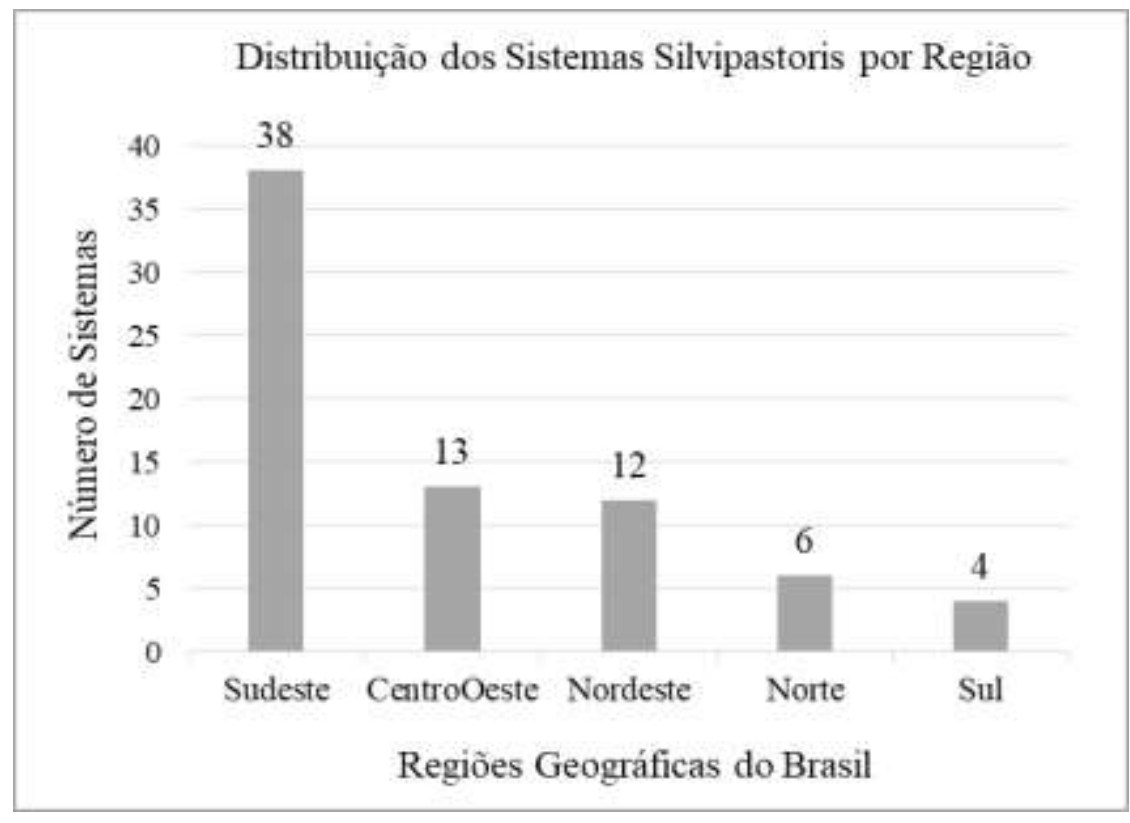

Fonte: Elaborado pelos autores.

A Figura 4 enaltece a disparidade na quantidade de artigos publicados entre as regiões geopolíticas brasileiras. O Sudeste contribui com $52 \%$ dos estudos, enquanto o Centro-Oeste $17 \%$, Nordeste 16,4, Norte $8 \%$ e Sul apenas 5,5\%.

A degradação ambiental na Região Sudeste é uma característica bastante comum, notada pela abundância de terras desmatadas e áreas degradadas (Balbino et al., 2011). Nesta região a adoção de SSPs tem avançado com rapidez como alternativa ao modelo vigente de produção. O maior número de pesquisas com SSPs concentra-se no Estado de Minas Gerais, onde também se concentram as atividades de reflorestamento com eucaliptos de empresas privadas do ramo da indústria papeleira e silvicultura (Garcia et al., 2003).

Até meados de 1960, as atividades agrícolas no cerrado eram limitadas à produção extensiva de gado de corte, o que acelerava os processos de degradação. Este fato estava relacionado majoritariamente à falta de tecnologia pra produção agropecuária em agroecossistemas tropicais (Balbino et al., 2011). Hoje é sabido que nos estados de Mato Grosso e Goiás, os SSPs são indicados para áreas degradadas e aquelas inaptas para lavoura mecanizada (Wruck et al., 2015). Essa região apresenta grande potencial na adoção dos SSPs, embora ainda pouco utilizado (Melotto et al., 2009).

A região Nordeste do Brasil apresenta a maior parte de seu território dentro do Bioma Caatinga, sendo $57 \%$ caracterizado como semiárido. Nesta região, predominam sistemas de 
produção desenvolvidos para pequenas propriedades familiares caracterizados em sua maioria pela economia de subsistência (Balbino et al., 2011). As palmeiras e coqueiros nativos adaptados às características da região têm sido utilizados nos SSPs como parte da cultura local. A título de exemplo, o babaçu é uma palmeira natural da região que se adaptou e é utilizada nestes sistemas, com agroflorestas plantadas e não plantadas (Araújo et al., 2016).

No Norte do país, o processo de ocupação humana recente está historicamente ligado a derrubada e queima da floresta Amazônica. Nos diversos estados que possuem território no Bioma Amazônia encontramos atividades de extração de recursos madeireiros, não madeireiros e criação de bovinos em destaque (Dias-Filho \& Ferreira, 2008; Fujisaka et al., 1996; Schmitz, 2007). Entretanto, a partir de 2004 iniciou-se o processo contínuo de redução dos desmatamentos e queimadas na Amazônia como resultado das medidas regulamentadas no Plano de Ação para Prevenção e Controle do Desmatamento na Amazônia Legal (Martínez et al., 2019). Assim, de 2004 até 2019, houve uma redução de mais de 64\% no desmatamento na região (Instituto De Pesquisas Espaciais, 2019).

Isso pode ter sido impulsionado pela flora diversa e abundante da região da Amazônia e pela na Lei $\mathrm{N}^{\mathrm{o}} 12.727$, de 17 de outubro de 2012, que aponta que todo imóvel rural deve manter área com cobertura de vegetação nativa a título de Reserva Legal (RL), o que na região chega a 80\%. Nesse sentido, a intensificação do uso da terra em áreas já antropizadas utilizando os sistemas de integração lavoura-pecuária-floresta tem sido adotado como uma das alternativas vigentes para a região ( Balbino et al., 2011; Martínez et al., 2019).

O sul do Brasil recebeu fortes interferências das migrações internas e da chegada dos imigrantes europeus. Assim originou-se um sistema agrário colonial diversificado, em pequenas propriedades, e com raiz europeia (Balbino et al., 2011; Etges \& Karnopp, 2020). A adoção de SSP nestas propriedades tem encontrado resistências culturais, e em geral está relacionada a políticas governamentais regionais, com objetivo encorajar ações de desenvolvimento socioeconômico atreladas às demandas das industriais locais (Ribaski, 2019). O setor de base florestal tem se beneficiado, já que somente no estado do Rio Grande do Sul são 368,4 mil empregos gerados (AGEFLOR, 2017). As empresas madeireiras da região tem fomentado o plantio do pinheiro (Pinus sp.), eucalipto (Eucalyptus sp.), e menos frequentemente a erva-mate (Ilex paraguariensis) e a bracatinga (Mimosa scabrella) (Nicodemo et al., 2015). 


\subsection{Diversidade do componente arbóreo nos sistemas silvipastoris}

Ao substituir os remanescentes florestais altamente diversificados, por monoculturas arbóreas ("florestas plantadas"), plantios agrícolas ou pastagens, alterações no ecossistema original modificam todos os atributos morfológicos, físicos, químicos e biológicos ali presentes (Jardim et al., 2019). A biodiversidade pode servir como importante fator indicativo da resiliência dos agroecossistemas, possibilitando a conservação da água e do solo, além da ciclagem de nutrientes e controle biológico de insetos e pragas (Altieri \& Nicholls, 2003; Prestes \& Vincenci, 2019). Deste modo, fica claro o potencial dos sistemas silvipastoris como importante ferramenta para reabilitação dos agroecossistemas e melhoria da diversidade em diferentes biomas, especialmente onde predominam as monoculturas arbóreas e pastagens (Lima \& Gama, 2018).

A integração simultânea das espécies arbóreas relaciona-se de forma sustentável e busca atender demandas do produtor rural, como alimento, madeira, lenha, frutas, plantas medicinais (Garcia et al., 2010), resina, látex, e óleos. Sinergicamente, os SSPs tem incrementado a biodiversidade (Rover et al., 2018; Simione et al., 2019), os atributos do solo e a ciclagem de nutrientes (Battisti et al., 2018). Também tem promovido à melhoria do microclima e ambiência (Deniz et al., 2019, 2020; Kretzer, 2019), o estoque de carbono nos elementos florestais e nas pastagens (Almeida Silva et al., 2020) e a produção forrageira (Almeida Silva et al., 2020; Kretzer, 2019), entre outros serviços ecossistêmicos (Schmitt Filho et al., 2013; Schmitt Filho \& Farley, 2020).

Na definição dos arranjos e modalidades dos Sistemas Silvipastoris, a melhor espécie será aquela que seja plenamente adaptada às condições dos agroecossistemas, que possui mercado garantido, e haja domínio dos tratos culturais (Behling et al., 2013). Em relação a diversidade dos componentes arbóreos, seja espécies lenhosas ou herbáceas de médio porte, os artigos indicam que $49 \%$ dos sistemas estudados se caracterizam como monoculturas, $18 \%$ apresentam apenas duas espécies, $8 \%$ apresentam três, e 25\% dos SSPs apresentam quatro ou mais espécies.

A região Norte se destaca pela alta diversidade dos componentes arbóreos, $50 \%$ dos SSPs apresentam mais do que três espécies arbóreas, enquanto a região Sudeste, somente $34 \%$. (Figura 5). 
Figura 5. Número de SSPs citados nos artigos científicos desta revisão, distribuídos pela diversidade do componente arbóreo. A cor cinza escuro representa o número de SSP por regiões geográficas, enquanto o cinza claro representa o número de sistemas com diversidade arbórea igual ou superior a três espécies.

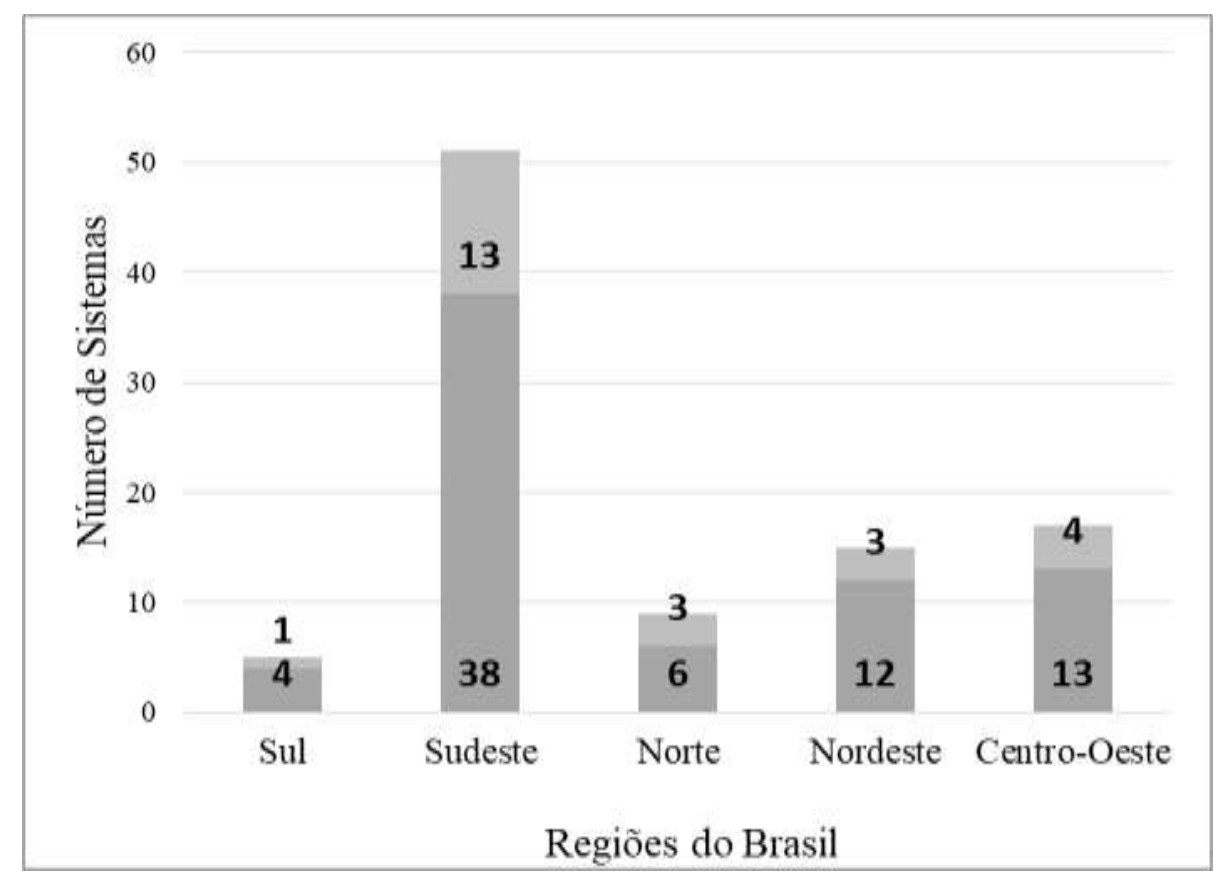

Fonte: Elaborado pelos autores.

Nota-se na Figura 5, a baixa diversidade dos componentes arbóreos nos sistemas silvipastoris em grande parte das regiões geográficas brasileiras. Poucos sistemas apresentam mais de três espécies em uma mesmo local, e raros são os SSPs com espécies nativas, principalmente no sul e sudeste do país.

A região Norte tem apresentado maior diversidade arbórea nos SSPs, o que pode estar relacionado ao contexto histórico de uso e ocupação do solo na região. Dentre os trabalhos avaliados por Veiga et al. (2000), 45\% dos sistemas estudados apresentavam três ou mais espécies arbóreas. Ainda de acordo com estes autores, na região utiliza-se SSPs com componentes arbóreos não plantados, em áreas em processo de desmatamento seletivo, ou com diferentes estágios de regeneração. As espécies nativas estão bastante presentes com potencial ainda não devidamente conhecido, e muitas vezes, com mercado não consolidado (Behling et al., 2013). Os avanços em pesquisas nestas áreas vêm demonstrando que o fornecimento de produtos florestais não madeireiros (PFNM) de espécies nativas é bastante promissor podendo viabilizar SSPs mais complexos, com melhor rentabilidade e integrados 
ao ecossistema (Trevisan et al.; 2019). A escolha de PFNM para compor SSP pode ser um fator-chave, pois envolve espécies adaptadas aos ecossistemas locais (Pott \& Pott, 2003).

Nas demais regiões, as espécies exóticas dominam na maioria dos SSPs reportados nos estudos avaliados. Isto ocorre, pois até recentemente estas eram as únicas espécies estudadas e fomentadas pelos institutos de pesquisa e extensão. Estas espécies exóticas também tem apresentado boa produtividade, tecnologia adaptada e mercado consolidado, apesar de controlado pelos grandes conglomerados agroindustriais (Behling et al., 2013). A produção de madeira, na maioria das vezes, tem sido a principal justificativa para o plantio de espécies exóticas nas propriedades rurais e em SSPs (Lima \& Gama, 2018).

O Brasil se destaca pelo plantio de "monoculturas arbóreas" ou "florestas" homogêneas, em especial de pinus e eucalipto. Em 2017, essas florestas totalizaram 9,431 milhões de hectares, sendo 78,5\% de eucalipto (Eucalyptus sp.) e 21,5\% de pins (Pinus sp.) (IBGE, 2017). Estes dados corroboram com os resultados encontrados por Paula et al. (2013) onde o gênero Eucalyptus sp. está presente em 57\% dos SSPs avaliados, e em $31 \%$ deles é o único componente arbóreo (Figura 6). Existem muitas justificativas para maior utilização destas duas espécies exóticas, tais como o fornecimento de madeira para usos múltiplos, fomento institucional, disponibilidade de mudas, pesquisa e conhecimento de silvicultura, existência de material genético melhorado, e mercado consolidado (Paula et al., 2013).

Figura 6. Distribuição das espécies de Eucalyptus dos Sistemas Silvipastoris dos artigos científicos selecionados.

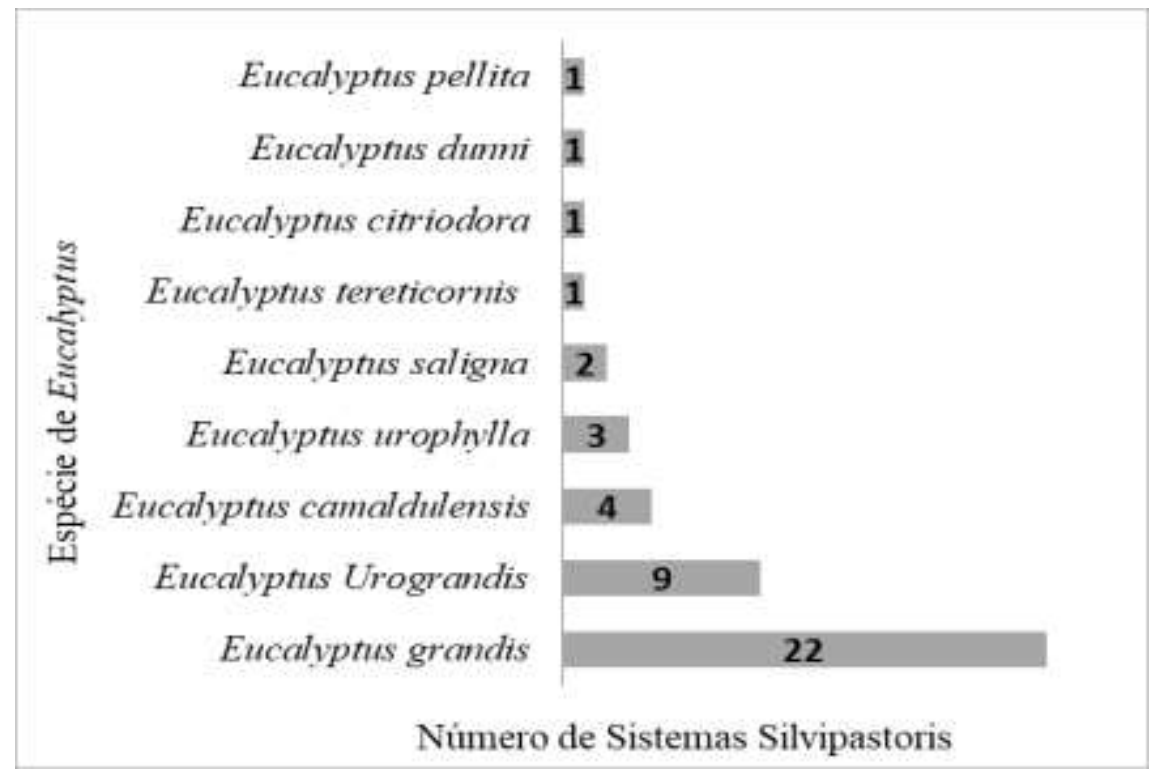

Fonte: Elaborado pelos autores. 
Research, Society and Development, v. 9, n. 10, e7019109016, 2020

(CC BY 4.0) | ISSN 2525-3409 | DOI: http://dx.doi.org/10.33448/rsd-v9i10.9016

Na Figura 6 observa-se a distribuição das espécies de Eucalyptus sp., com destaque para o Eucalyptus grandis nos SSPs avaliados nesta revisão sistemática.

O eucalipto (Eucalyptus sp.) tem apresentado vantagens competitivas em relação a espécies nativas, devido ao fomento institucional e industrial, com mais de um século de melhoramento genético, produção de híbridos e clonagem. Entretanto, a dependência de um pequeno número de variedades e a perda do patrimônio genético, representa uma grande ameaça aos sistemas ecológicos, à integridade da biodiversidade e aos Biomas Brasileiros. Estes Biomas sãos responsáveis pelos serviços ecossistêmicos essenciais para a população brasileira, e inclusive para a própria agropecuária (Altieri, 1999). A sustentabilidade dos SSPs constituídos pelo componente animal, gramíneas e eucaliptos necessita de maiores estudos, pois é possível que os desserviços ambientais se sobrepujem aos serviços ecossistêmicos (Battisti et al., 2018; Bernardino \& Garcia, 2009). Na introdução das espécies para os SSPs é importante que seja mantida a diversidade funcional do componente arbóreo, de modo que sinergicamente disponibilizem recursos bióticos e abióticos adequados para atender as exigências dos demais componentes (Bernardino \& Garcia, 2009).

\subsection{Lacunas de pesquisa}

Esta revisão expõe alguns descompassos e lacunas no repertório de publicações relacionadas aos SSPs brasileiros, verificando-se que a maior parte é direcionada ao componente animal, 28\% do total dos artigos. Existe uma crescente preocupação com boas práticas de manejo animal associadas a estudos do microclima, ambiências, comportamento e bem-estar, pois são fatores que influenciam diretamente na produtividade e sustentabilidade dos sistemas de produção.

A distribuição dos demais trabalhos se concentra no componente arbóreo, com $26 \%$ das publicações e forragem com $23 \%$. Os aspetos menos estudados são os elementos do solo com $14 \%$ e SSPs de maneira geral com 3\% (Figura 7). 
Figura 7. Elementos temáticos identificados como objeto de estudo nos trabalhos avaliado, representado ou não no objetivo do artigo descrito.

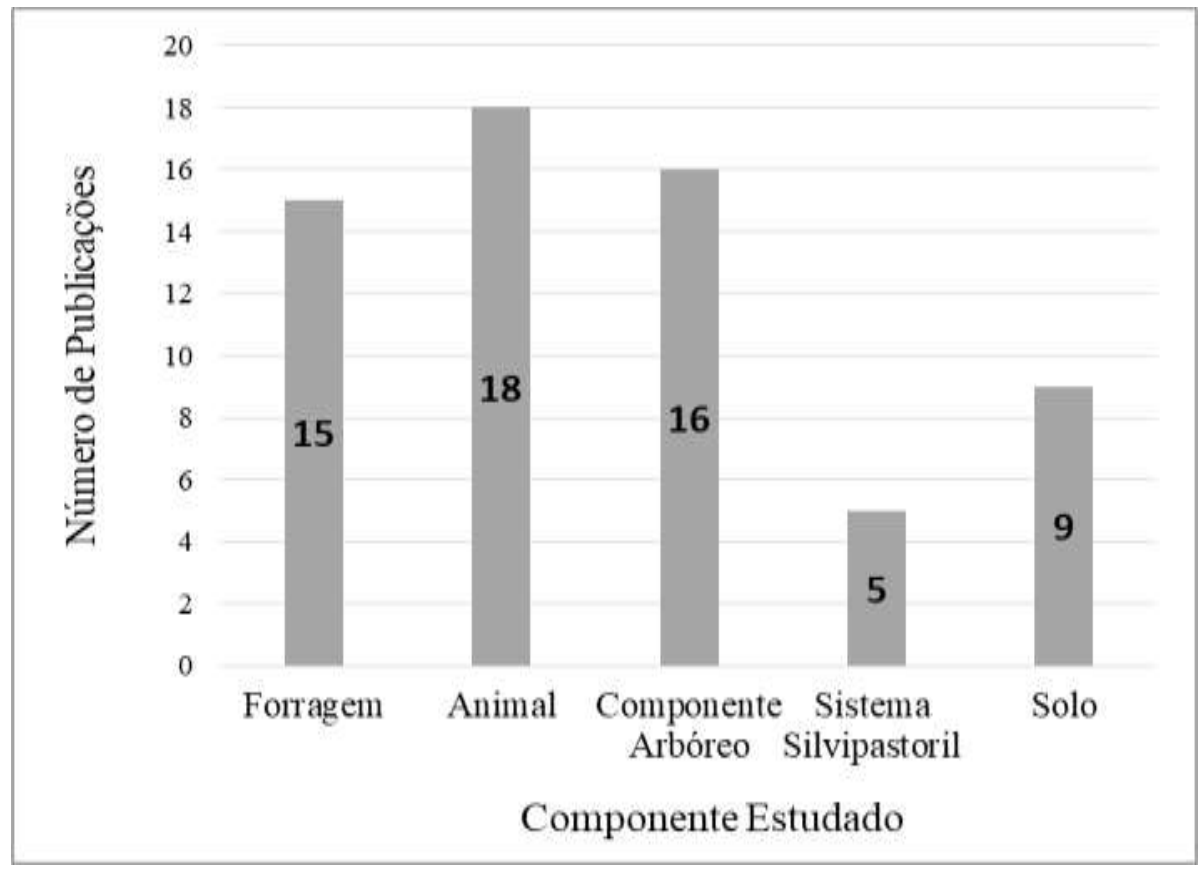

Fonte: Elaborado pelos autores.

$\mathrm{Na}$ Figura 7 esta caracterizado os eixos temáticos dos artigos avaliados, com concentração em 3 áreas majoritarias.

Dentro de cada um destes componentes, os objetivos vão se mostrando presentes em maiores detalhes, auxiliando a identificação do conhecimento sobre o assunto. Para o componente animal, o comportamento predomina nos estudos, apresentando informações relacionadas ao bem-estar e ambiência (Schütz et al., 2010). Para forragem, muito se discute sobre produtividade e qualidade (Araújo et al., 2016; Santos et al., 2016). Sobre o componente arbóreo, a maior parte dos estudos ressalta a dificuldade que ainda persiste em criar delineamentos florestais adequados para sistemas silvipastoris que contemplem a multitude de ecossistemas, Biomas e a marcante biodiversidade florística brasileira (Paula et al., 2013) (Figura 8). 
Figura 8. Classificação dos subtópicos de pesquisa em cada uma das grandes áreas avaliadas.

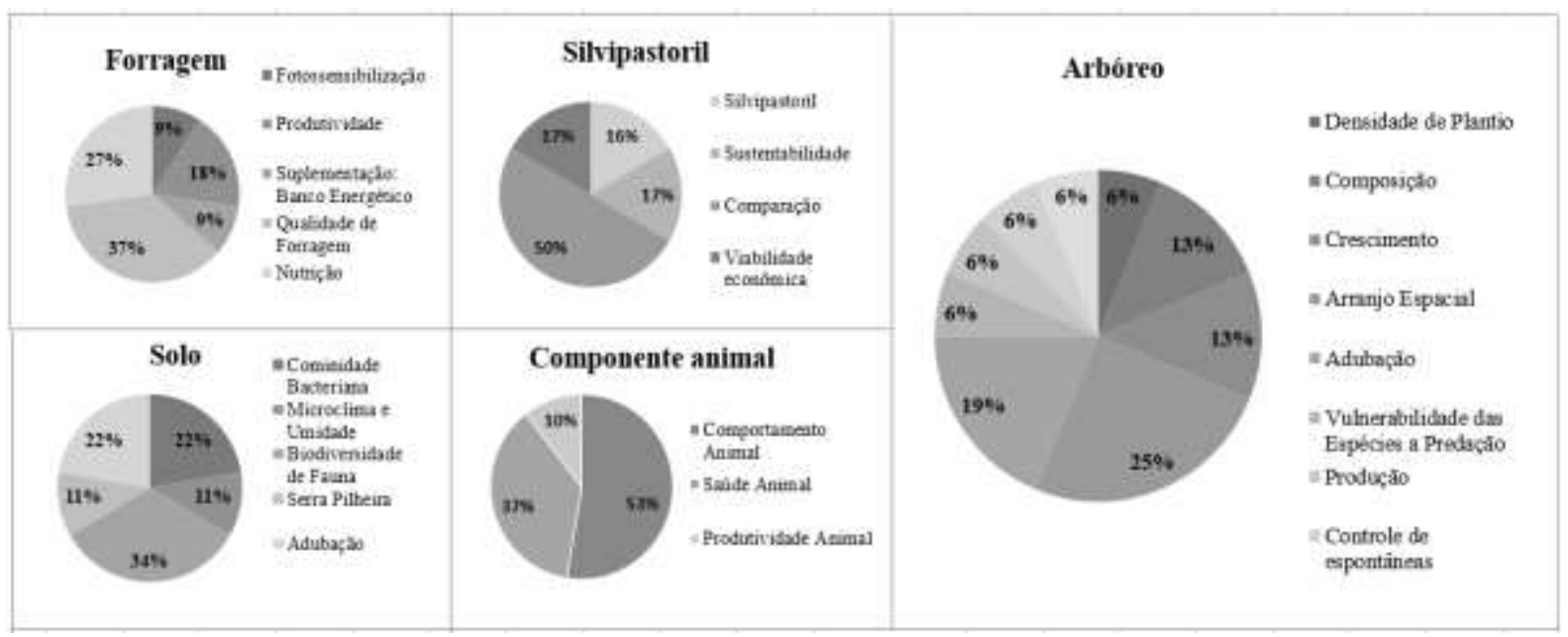

Fonte: Elaborado pelos autores.

A Figura 8 evidencia a concentração dos trabalhos científicos em poucas subáreas de cada uma das cinco grandes áreas. Destas cinco grandes áreas, três estão fortemente interligadas nos agroecossistemas e SSPs. A sombra produzida pelas árvores modifica o microclima (Deniz et al., 2019, Kretzer, 2019) influenciando a quantidade e qualidade da forragem disponível (Almeida Silva et al., 2020), sendo associadas às melhores condições de conforto térmico para os animais (Deniz et al., 2020) e indicando um possível aumento no consumo de forragem e ganho de peso de animais em pastejo (Paciullo et al., 2014). Geralmente, gramíneas forrageiras sombreadas tendem a apresentar maior teor de proteína bruta e digestibilidade da matéria seca (Almeida Silva et al., 2020). A discussão do incremento de biodiversidade nos SSPs ainda é contida aos aspectos biofísicos e in loco, e poucos extrapoladas para a visão sistêmica dos habitats do entorno e da paisagem (Schmitt Filho \& Farley, 2020; Simione et al., 2019).

Este tipo de análise dos componentes nos permite criar um mapa de Lacuna, que consiste no cruzamento dos elementos temáticos e suas evidências de estudo. O objetivo é facilitar a visualização da frequência com que cada relação entre duas variáveis foi evidenciada nos artigos, deixando claras as lacunas de pesquisa de cada área.

O mapa ilustra, através de uma escala da intensidade de cores (heatmap scale), como a densidade e lacunas de evidências estão distribuídas entre os fatores analisados (Figura 9). 
Figura 9. Mapa de Lacunas de Evidências (Evidence Gap Map, EGM).

\begin{tabular}{|c|c|c|c|c|c|c|c|c|c|c|}
\hline & & & ausas & & & & & & & \\
\hline Forragem & Anii & & Arbóreo & & & & opast & & & \\
\hline & C.A & S.A & & $\mathrm{Fa}$. & $\mathrm{Fe}$. & Su. & Co. & Ec. & & \\
\hline 5 & 9 & 5 & 9 & 0 & 1 & 0 & 0 & 0 & & Arbóreo \\
\hline 1 & 0 & 0 & 5 & 2 & 2 & 0 & 0 & 0 & Fauma & Solo \\
\hline 5 & 0 & 0 & 5 & 4 & 1 & 0 & 0 & 0 & Fertilidade & \\
\hline 1 & 0 & 0 & 0 & 0 & 0 & 0 & 0 & 0 & Sustentabilidade & Silvipastonl \\
\hline 3 & 0 & 3 & 2 & 1 & 0 & 0 & 2 & 0 & Comparativo & \\
\hline 1 & 0 & 0 & 0 & 0 & 0 & 0 & 0 & 0 & Ecónomico & \\
\hline 2 & 0 & 0 & 1 & 0 & 0 & 0 & 0 & 0 & Saude Animal & Animal \\
\hline 5 & 1 & 0 & 0 & 0 & 0 & 0 & 0 & 0 & Comportamerto Animal & \\
\hline 7 & 2 & 2 & 2 & 1 & 0 & 0 & 0 & 0 & & Forragem \\
\hline
\end{tabular}

Fonte: Elaborado pelos autores.

A Figura 9 evidencia que o componente causal arbóreo e forragem são os principais impulsores do desenvolvimento dos SSPs, devido ao maior número de relações com as consequências encontradas nas publicações. Enquanto, o componente econômico apresenta a maior lacuna de conhecimento sobre o tema no Brasil.

Foram catalogados poucos artigos estudando questões econômicas SSPs, principalmente quando associado a espécies nativas, PFNM ou desenhos alternativos de SSPs. Infelizmente, ainda a área de econômica não tem se debruçado sobre as dinâmicas financeiras da implantação, gestão e competitividade microeconômica dos SSPs. A lacuna se faz ainda maior quando se migra para economia ecológica, com avaliações relacionadas aos serviços ecossistêmicos e desserviços ambientais. Esta revisão demonstra que ainda existem muitos questionamentos sobre a temática dos SSPs, evidenciando grandes as lacunas em áreas absolutamente relevantes.

\section{Considerações Finais}

As Revisões Sistemáticas são essenciais para avaliar as evidências sobre o que é conhecido, sintetizá-las e formular prioridades de pesquisas futuras. A relevância desta análise sistemática está relacionada ao mapeamento do "estado da arte" das pesquisas sobre os sistemas silvipastoris no Brasil (Bernardino \& Garcia, 2009)

Dentro de uma perspectiva histórica, os SSPs se caracterizam uma tecnologia recente no Brasil. A Empresa Brasileira de Pesquisa Agropecuária e as instituições estaduais tem um 
papel importante no fomento e divulgação destes resultados de pesquisas e técnicas nas diversas regiões geopolíticas brasileiras.

Observa-se que os artigos tendem a abordar com maior frequência o viés mais biofísico dos SSPs. Muitos benefícios decorrentes dos SSPs estão relacionados aos serviços ecossistêmicos associados à restauração e reabilitação ecológica, conservação ambiental, e até serviços de provisão com melhorias na rentabilidade. Os serviços ecossistêmicos são os benefícios que o homem obtém dos ecossistemas. Pouco se pontua, na multiplicidade de serviços ecossistêmicos de provisão, regulação, suporte e culturais que advém do enriquecimento da biodiversidade dos SSPs (Palm et al., 2014). Mostra-se necessário incorporar metodologias interdisciplinares que abordem de forma transversa e eficaz as distintas dimensões socioambientais e econômicas relacionadas aos SSPs, incluindo análises dos incrementos da biodiversidade.

Hoje, mais do que nunca passou a ser premente o desenvolvimento e estudo de SSPs com espécies da flora local que viabilizem a reabilitação dos agroecossistemas e restauração ecológica dos Biomas, que sejam culturalmente atraentes e economicamente competitivos.

Sistemas de produção que mitiguem o impacto ambiental da agricultura convencional enquanto economicamente competitivos parecem ser a necessidade mor neste início de década. Os SSPs em muito podem atender esta demanda, todavia grandes lacunas de pesquisa devem ser atendidas, inclusive política e institucionalmente. Neste sentido, sugerimos que pesquisas futuras relacionadas aos SSPs perpassem os desenhos alternativos com espécies arbóreas nativas, os sistemas biodiversos com PFNM, a sociobiodiversidade, a restauração ecológica, o potencial dos SSPs na reabilitação dos agroecossistemas, a microeconomia, e inclusive a economia ecológica considerando os serviços ecossistêmicos e desserviços ambientais.

\section{Agradecimentos}

Este estudo foi financiado pelo Conselho Nacional de Desenvolvimento Científico e Tecnológico-CNPq através do projeto "Analyzing Ecosystem Services from Agroecology in the Atlantic Forest: a Participatory Modeling Approach (PVE / CNPq 71/2015)" no âmbito do Programa de Pós-graduação em Ciência Ambiental da Universidade de São Paulo (PROCAM/USP), do Laboratório de Sistemas Silvipastoris e Restauração Ecológica LASSre/UFSC, e do Programa de Pós-graduação em Agroecossistemas da Universidade 
Federal de Santa Catarina PPGA/UFSC. O Estudo contou com o apoio da Coordenação Brasileira de Aperfeiçoamento de Pessoal de Nível Superior CAPES/MEC e do Gund Institute for Environment da University of Vermont GUND IE/UVM EUA.

\section{Referências}

Abadias, M. I., Fonseca, P. R. B., \& Barbos, C. H. (2020). Manejo Da Pecuária: Uma Análise Sobre Impactos Ambientais. Revista EDUCAmazônia - Educação Sociedade e Meio Ambiente, Humaitá, 13, 113-125.

AGEFLOR. (2017). A indústria de base florestal no RS: dados e fatos. www.ageflor.com.br

Almeida Silva, A. de, Schmitt Filho, A. L., Kazama, D. C. da S., Loss, A., Souza, M., Piccolo, M. de C., Farley, J., \& Sinisgalli, P. A. de A. (2020). Estoques de carbono e nitrogênio no Sistema Silvipastoril com Núcleos: a nucleação aplicada viabilizando a pecuária de baixo carbono. Research, Society and Development, 9(9). https://doi.org/10.33448/rsdv9i10.8589

Altieri, M. A. (1999). The ecological role of biodiversity in agroecosystems. Invertebrate Biodiversity as Bioindicators of Sustainable Landscapes, 19-31. https://doi.org/10.1016/b 978-0-444-50019-9.50005-4

Altieri, M. A., \& Nicholls, C. I. (2003). Soil fertility management and insect pests: Harmonizing soil and plant health in agroecosystems. Soil and Tillage Research, 72(2), 203211. https://doi.org/10.1016/S0167-1987(03)00089-8

Alves, F. V., Laura, V. A., \& de Almeida, R. G. (2015). Sistemas agroflorestais: a agropecuária sustentável. Embrapa Gado de Corte-Livro técnico (INFOTECA-E).

Antonangelo, A., \& Bacha, C. J. C. (1998). As Fases da Silvicultura no Brasil. Revista Brasileira de Economia, 52(1), 207-238.

Araújo, R. A. de, Rodrigues, R. C., Costa, C. dos S., Sousa, F. N., Santos, F. O., Costa, A. J. T. de L., Silva, I. R. da, \& Rodrigues, M. M. (2016). Composição químico-bromatológica e 
Research, Society and Development, v. 9, n. 10, e7019109016, 2020

(CC BY 4.0) | ISSN 2525-3409 | DOI: http://dx.doi.org/10.33448/rsd-v9i10.9016

degradabilidade in situ de capim- Marandu em sistemas silvipastoris formados por babaçu e em monocultivo. Revista Brasileira de Saúde e Produção Animal, 19(3), 347-359. https://doi.org/10.1590/s1519-99402018000300011

Balbino, L. C., Cordeiro, L. A. M., Porfírio-da-Silva, V., Moraes, A. D., Martínez, G. B., Alvarenga, R. C., \& Galerani, P. R. (2011). Evolução tecnológica e arranjos produtivos de sistemas de integração lavoura-pecuária-floresta no Brasil. Pesquisa Agropecuária Brasileira, 46(10). https://doi.org/10.1590/s0100-204x2011001000001

Balbino, L. C., Barcellos, A. D. O., \& Stone, L. F. (2011). Marco referencial: integração lavoura-pecuária-floresta. Embrapa Cerrados-Livro científico (ALICE).

Battisti, L. F. Z., Schmitt Filho, A. L. S., Loss, A., \& de Almeida Sinisgalli, P. A. (2018). Soil chemical attributes in a high biodiversity silvopastoral system. Acta Agronomica, 67(4), 486493. https://doi.org/10.15446/acag.v67n4.70180

Behling, M., Pedreira, B. C. E., Carnevalli, R. A., Lopes, L. B., \& Tonini, H. (2013, maio). Pastagens: Planejamento da arborização de pastagens para produção de Leite. Revista Leite Integral, 14-21.

Bernardino, F. S., \& Garcia, R. (2009). Sistemas Silvipastoris. Pesquisa Florestal Brasileira, 60, 77-87. https://doi.org/10.4336/2009.pfb.60.77

Broom, D. M. (2017). Components of sustainable animal production and the use of silvopastoral systems. Revista Brasileira de Zootecnia, 46(8), 683-688. https://doi.org/10.1590/S1806-92902017000800009

Cooper, H., \& Hedges, L. V. (2009). The Handbook of Research Synthesis and Meta-Analysis (3-17).

Deniz, M., Schmitt Filho, A. L., Hötzel, M. J., de Sousa, K. T., Pinheiro Machado Filho, L. C., \& Sinisgalli, P. A. (2020). Microclimate and pasture area preferences by dairy cows under high biodiversity silvopastoral system in Southern Brazil. International Journal of Biometeorology, 1-11. https://doi.org/10.1007/s00484-020-01975-0 
Deniz, M., Schmitt Filho, A.L., Hoetzel, M.J., Farley, J. Quadros, S.F. (2018). High Biodiversity High biodiversity silvopastoral system as an alternative to improve the thermal environment in the dairy farms. International Journal of Biometeorology, 63:8392. DOI: 10.1007/s00484-018-1638-8.

Dias-Filho, M. B., \& Ferreira, J. N. (2008). Barreiras à adoção de sistemas silvipastoris no Brasil. Embrapa Amazônia Oriental-Documentos (INFOTECA-E).

Etges, V. E., \& Karnopp, E. (2020). A agroindústria familiar no contexto do sistema agrário colonial no Sul do Brasil. Redes - Revista do Desevolvimento Regional, 25(1), 268-283. https://doi.org/10.17058/redes.v25i1.14255

Fujisaka, S., Bell, W., Thomas, N., Hurtado, L., \& Crawford, E. (1996). Slash-and-burn agriculture, conversion to pasture, and deforestation in two Brazilian Amazon colonies. Agriculture, Ecosystems and Environment, 59(1-2), 115-130. https://doi.org/10.1016/01678809(96)01015-8

Furtado, R. C., Abreu, L. S. de, \& Furtado, A. T. (2019). Sistemas agroflorestais. Cadernos de Ciência \& Tecnologia, 35(3), 427-451. https://doi.org/10.35977/01041096.CCT2018.V35.26319

Garcia, R., Tonucci, R. G., \& Gobbi, K. F. (2010). Sistemas silvipastoris: uma integração pasto, árvore e animal. Sistema Agrossilvipastoril Integração lavoura, pecuária e floresta. Viçosa, MG: Sociedade de Investigações Florestais, 123-166.

Garcia, R., Couto, L., Andrade, C. M. S., \& Tsukamoto Filho, A. A. (2003). Sistemas Silvipastoris na região Sudeste: A Experiência da CMM. Seminário de Sistemas Agroflorestais e Desenvolvimento Sustentável: Opção de sustentabilidade para áreas tropicais e subtropicais, 173-187.

Huntsinger, L., Sulak, A., Gwin, L., \& Plieninger, T. (2004). Oak Woodland Ranchers in California and Spain. In Sustantability and Management 309-326. 
Instituto Brasileiro de Geografia e Estatística. IBGE. (2017). IBGE | Portal do IBGE | IBGE. https://www.ibge.gov.br/

Instituto De Pesquisas Espaciais. (2019). PRODES. Coordenação-Geral de Observação da Terra. Recuperado de http://www.obt.inpe.br/OBT/assuntos/programas/amazonia/prodes

Jardim, M. de S. S., Ferraz, A. M. da R., Simões, V. J. L. P., Izidro, J. L. P. S., \& Leite, M. L. de M. V. (2019). Sistemas silvipastoris: meio ambiente, produção e qualidade de forragem e resposta animal. Brazilian Journal of Applied Research \& Agrotechnology, 12(3), 135-143. https://doi.org/10.5935/paet.v6.n1.13

Joseph, L., Schmitt Filho, A. L., Sinisgalli, P. A., Farley, J., Zambiazi, D. C. (2019) Sistemas silvipastoris e serviços ecossistêmicos: a visão dos produtores de leite da capital da agroecologia no Sul do Brasil. Revista de Ciências Agrárias, 42 (3): 829-841. https://doi.org/10.19084/rca.17116

Kretzer, S. G. (2019). Influência do Sistema Silvipastoril com Núcleos de alta diversidade na dinâmica e microclima forrageiro. Orietador, Schmitt Filho, A. L., Coorientador, Kazama, D. Dissertação (Mestrado em Agroecossistemas) Programa de Pos-Graduação, Agroecosistemas, Universidade Federal de Santa Catarina. Florianópolis, p. 82.

Lima, M. C. D. de, \& Gama, D. C. (2018). O Sistema De Integração Lavoura-PecuáriaFloresta No Brasil: Conceitos, Desafios E Novas Perspectivas. Agroforestalis News, 3(1), $31-51$.

Martinez, G., De Azevedo, C. M. B. C., Silva, A., Botelho, F., De Oliveira, T. K., \& Godinho, V. (2019). Integração lavoura pecuária floresta na região Norte do Brasil. Embrapa Amazônia Oriental-Capítulo em livro científico (ALICE).

Melotto, A., Nicodemo, M. L., Bocchese, R. A., Laura, V. A., Neto, M. M. G., Schleder, D. D., Pott, A., \& da Silva, V. P. (2009). Survival and initial growing of native tree seedlings in pastures of central Brazil. Revista Árvore, 33(3), 425-432. https://doi.org/10.1590/s010067622009000300004 
Nicodemo, M. L. F., Silva, V. P. da, Thiago, L. R. L. de S., Gontijo N., M. M., \& Laura, V. A. (2015). Sistemas silvipastoris - introdução de árvores na pecuária do Centro-Oeste brasileiro. Embrapa Gado de Corte - Documentos, 146. Campo Grande, MS: Embrapa Gado de Corte, 2004.

Paciullo, D. S. C., Pires, M. F. A., Aroeira, L. J. M., Morenz, M. J. F., Maurício, R. M., Gomide, C. A. M., \& Silveira, S. R. (2014). Sward characteristics and performance of dairy cows in organic grass-legume pastures shaded by tropical trees. Animal, 8(8), 1264-1271. https://doi.org/10.1017/S1751731114000767

Palm, C., Blanco-Canqui, H., DeClerck, F., Gatere, L., \& Grace, P. (2014). Conservation agriculture and ecosystem services: An overview. Agriculture, Ecosystems and Environment, 187, 87-105. https://doi.org/10.1016/j.agee.2013.10.010

Paula, R. R., Reis, G. G., Reis, M. G. F., Oliveira Neto, S. N., Leite, H. G., Melido, R. C. N., Lopes, H. N. S., \& Souza, F. C. (2013). Eucalypt growth in monoculture and silvopastoral systems with varied tree initial densities and spatial arrangements. Agroforestry Systems, 87(6), 1295-1307. https://doi.org/10.1007/s10457-013-9638-5

Peri, P. L., Dube, F., \& Varella, A. (2016). Silvopastoral Systems in Southern South America $11^{\mathrm{o}} \mathrm{ed}$, Vol. 11. https://doi.org/10.1007/978-3-319-24109-8

Pott, A., \& Pott, V. J. (2003). Plantas Nativas Potenciais para Sistemas Agroflorestais em Mato Grosso do Sul. Seminário Sistemas Agroflorestais E Desenvolvimento Sustentável.

Prestes, R. M., \& Vincenci, K. L. (2019). Bioindicator as Environmental Impact Assessment. Brazilian Journal of Animal and Environmental Research Braz. J. Anim. Environ. Res, 2(4), 1473-1493.

Ribaski, J. (2019). Avaliação do desempenho silvicultural e econômico de cinco materiais genéticos de Eucalyptus em sistema silvipastoril, no bioma Pampa do Rio Grande do Sul. Embrapa Florestas-Comunicado Técnico (INFOTECA-E).

Rover, C. M., Schmitt Filho, A. L., Simioni, G. F., Fettuccia, D., \& Sinisgalli, P. A. (2018). 
Percepção de estudantes sobre a biodiversidade do solo Students perception on soil biodiversity. Cadernos de Agroecologia, 13(1).

Saibro, J. C. de, \& Garcia, R. (2005). Sistemas silvipastoris integram árvores, pastos e animais. Visão agricola, 3, 94-96.

Santos, D. de C., Guimarães Júnior, R., Vilela, L., Pulrolnik, K., Bufon, V. B., \& França, A. F. de S. (2016). Forage dry mass accumulation and structural characteristics of Piatã grass in silvopastoral systems in the Brazilian savannah. Agriculture, Ecosystems and Environment, 233, 16-24. https://doi.org/10.1016/j.agee.2016.08.026

Schmitt Filho, A., Farley, J., Alvez, J., Alarcon, G., \& Rebollar, P. M. (2013). Integrating Agroecology with Payments for Ecosystem Services in Santa Catarina's Atlantic Forest. In Governing the Provision of Ecosystem Services 333-355. https://doi.org/10.1007/978-94007-5176-7_17

Schmitt Filho, A. L., \& Farley, J. (2020). Transdisciplinary case study approaches to the ecological restoration of rainforest ecosystems. In Ecological Economic and Socio Ecological Strategies for Forest Conservation: A Transdisciplinary Approach Focused on Chile and Brazil (p. 185-212). Springer International Publishing. https://doi.org/10.1007/978-3-03035379-7_10

Schmitz, H. (2007). A Transição da agricultura Itinerante na Amazônia para novos sistemas. Revista Brasileira de Agroecologia, 2(1), 46-59.

Schreiner, H. G. (1983). Área experimental silvipastoril no sul do Paraná. EMBRAPAURPFCS.

Schütz, K. E., Rogers, A. R., Poulouin, Y. A., Cox, N. R., \& Tucker, C. B. (2010). The amount of shade influences the behavior and physiology of dairy cattle. Journal of Dairy Science, 93(1), 125-133. https://doi.org/10.3168/jds.2009-2416

Silva, T. L. A. da S. (2016). A apropriação captalista da silvicultura no Brasil e sua lógica de produção. Terra Livre, 1(50), 159-199. 
Simione, G. F., Schmitt Filho, A. L., Joner, F., Fantini, A. C., Farley, J., \& Moreira, A. T. (2019). Variação da assembleia de aves em áreas pastoris e remanescentes florestais adjacentes. Revista de Ciências Agrárias, 42(4), 884-895. https://doi.org/10.19084/rca.17601

Trevisan, A.C.D., Abreu, A.M., Nicolau V.R.V., Fantini, A.C., Schmitt Filho, A.L. (2019)

Quintias agroflorestais para a produção de frutos de Juçara em Santa Catarina. Revista Brasileira de Agroecologia, 14(4):102-112.https://doi.org/10.33240/rba.v14i4.23067

Veiga, J. B., Alves, C. P., Marques, L. C. T., \& da Veiga, D. F. (2000). Sistemas silvipastoris Amazônia Oriental. Embrapa Amazônia Oriental-Documentos (INFOTECA-E).

Wruck, F. J., Behling, M., \& Antonio, D. B. A. (2015). Sistemas Integrados em Mato Grosso e Goiás. In Sistemas agroflorestais: a agropecuária sustentável, 169-194.

\section{Porcentagem de contribuição de cada autor no manuscrito}

Giovana Pittarelli Bento - 37\%.

Abdon Luiz Schmitt Filho - 33\%.

Marcia Regina Faita - 30\%. 